\title{
Diagnosis and management of ectopic pregnancy
}

\author{
Catalina Diana STANICA, Adrian NEACSU, Romina Marina SIMA, Raluca Gabriela IOAN \\ Department of Obstetrics and Gynecology, "Carol Davila" University of Medicine and Pharmacy, \\ Bucharest, Romania
}

\begin{abstract}
Recently, substantial progress has been made in diagnosis and treatment, complicated ectopic pregnancy is a not negligible cause of maternal mortality, being the leading cause of maternal death in the first trimester of pregnancy and responsible for $6 \%$ of maternal deaths.

The aim of this study is to evaluate patients with ectopic pregnancy from a clinical, paraclinical and therapeutic point of view and to compare the influence of the management on hospitalization days and the preservation of patients fertility.

This paper presents a prospective case-control study for 65 patients, who have been diagnosed serologically and ultrasonically. In the group of patients studied, 25\% patients were treated with methotrexate, $65 \%$ were required surgical treatment, $4 \%$ required surgical and medical treatment, and $2 \%$ the management of expectation.

Laparoscopy significantly reduces the hospitalization period, contributing decisively to the rapid reintegration into society, increasing patient comfort, an excellent mental tone that promotes earlier healing, the number of complications decreases.
\end{abstract}

Keywords: ectopic pregnancy, methotrexate, salpingectomy

\section{INTRODUCTION}

Complications resulting from voluntary termination of pregnancy, infections, as well as postabortion endometritis can cause irreversible morphological lesions that favor, in the future, the occurrence of ectopic pregnancy $(1,2)$.

Abdominal surgery as well as cesarean operations result in the appearance of intra-abdominal adhesions that can change the tubal anatomy, favoring the appearance of ectopic pregnancies (3).

Immunological tests in conjunction with ultrasound (transabdominal and transvaginal) can make the diagnosis very accurately.

All women in the reproductive period who have the Crossen triad (abdominal pain, metrorrhagia and amenorrhea) should undergo paraclinical tests to confirm or refute the diagnosis of ectopic pregnancy (4).

The gestational sac, the first structure that can be visualized, is in the form of a thin echogenic ring, in the middle of which is the trophoblastic decidual reaction around the chorionic sac, structures that show an embryonic development detected later $(5,6)$. The pseudosack is a collection of fluid inside the uterine cavity. It is formed by a hemorrhage of the decidualized endometrium and is frequently associated with an ectopic pregnancy (20-44\%). It should not be confused with a normal pregnancy. The true gestational sac is located eccentrically, subendometrially, while the pseudosack fills the endometrial cavity. The diagnostic method is not $100 \%$ efficient, it is corroborated with the other specific investigations. 
The dosage of $\beta \mathrm{HCG}$, quantitative and dynamic, confirms the existence of pregnancy, the values doubling every 48 hours during the evolution of normal pregnancy. Serum $\beta$ HCG levels should increase by at least $66 \%$ in two days. An increase below this value is associated with an abnormally evolving intrauterine pregnancy or an ectopic pregnancy. It should be noted that there are exceptions: normal pregnancies (15\%) in which the dynamics of $\beta$ HCG do not follow the rule (increases $<66 \%$ ) and ectopic pregnancies (13\%) in which the $\beta$ HCG curve is identical to that of a normal pregnancy. Thus, $\beta$ HCG dosing may suggest the diagnosis, but may not support it, requiring further investigation. In conclusion, it can be stated that BHCG dosing is a sensitive method, but with a low specificity $(7,8)$.

Curative behavior includes expectation management, medical treatment and surgical therapy (9). In recent years, one method used successfully in the treatment of ectopic pregnancy in combination with methotrexate is the embolization of uterine arteries. Practicing the selective embolization of the uterine arteries, the method is used in the treatment of cervical and abdominal pregnancy, inserted at the scar after caesarean section but also tubal, having the advantage of maintaining the permeability of the fallopian tube, while preserving fertility (10-12).

As a mechanism of action, in ectopic pregnancy, methotrexate prevents the proliferation of cytotrophoblast cells, reduces cell viability and synthesis of $\beta \mathrm{HCG}$ and implicitly progesterone, causing resorption of ectopic pregnancy and tissue remodeling $(13,14)$.

From the point of view of surgery, the treatment is classified into classic and laparoscopic interventions, and from the point of view of the integrity of the female genital tract, the interventions can be conservative (linear salpingostomy and tubal evacuation of the conception product.

Hypersmolar glucose injections in tubal ectopic pregnancies have been studied, but they have significantly higher failure rates than standard medical or surgical management (15).

The great surgical emergency in gynecology is represented by the peritoneal flood which has as main manifestation the sudden pain, of very high intensity ("in the dagger"), followed by lipothymia, acute anemia and sometimes signs of hemorrhagic shock.

The study aims to compare how diagnostic and treatment methods can influence the evolution of patiens - complications, days of hospitalization, fertility conservation.

\section{MATERIAL AND METHOD}

This paper presents a prospective case-control study, conducted in the Bucharest Maternity Hospital Bucharest during 2018.
During the study, 65 patients with ectopic pregnancy were hospitalized. The diagnosis was established on the basis of clinical examination and specialized investigations, in accordance with current protocols.

Several parameters were evaluated: clinical signs, complications, paraclinical diagnostic methods used, treatment, length of hospitalization of laparoscopically versus classically operated patients (laparotomized).

Inclusion criteria were:

- Pregnancy test positive;

- Transvaginal ultrasound that can show the absence of images of intra or extrauterine sac, or extrauterine ovarian sac with or without embryonic elements, or equivocal intrauterine ultrasound images (endometrial fluid slide, false ovarian sac, ovarian sac without embryonic elements, paraovarian adnexal tumor mass which may present, but not obligatorily, ovarian sac +/embryonic elements)

- Deration of serum $\beta$ HCG in dynamics, at 48 hours;

- Hemodynamic stability;

- Hospitalized patients;

- Treatment-compliant patients.

All data were collected from hospitalization sheets and have been processed using Microsoft Excel. All data obtained were processed while maintaining the confidentiality of patients' personal data. Every patient that was enrolled in our study signed an informed consent, and the study was approved by the Ethical Committee.

\section{RESULTS}

Clinical manifestations found in patients in the study group were: amenorrhea, vaginal bleeding, abdominal pain (with colic, located in the lower abdomen or in an iliac fossa, often at a fixed point), lipothymia (in pregnancy complicated by hemoperitoneum), other signs: rectal tenesmus, pollakiuria, swelling of the breasts, digestive phenomena (nausea, vomiting), palpable laterouterine formation at the gynecological examination.

Pain was the most constant sign, being found in over $90 \%$ of cases (60 patients), accompanied by vaginal bleeding in over $78 \%$ of cases (51 patients), and amenorrhea was found in $35 \%$ of cases (23 patients). The location of the pain was located in most cases in the iliac fossae $75 \%$ (49 patients), but also in the hypogastrium (7\% of cases), lumbar region and diffuse pain in the lower abdominal floor (18\% of cases).

In descending order of frequency, the next clinical sign was vaginal bleeding, either menstrual or Pozzi distilling bleeding (black blood, chocolate-like), but there were cases in which it was absent. Out of the to- 
tal number of patients, $78 \%$ had hemorrhages (51 patients), of which 58\% were Pozzi distillatory hemorrhages and $20 \%$ menstrual blood hemorrhages, and $22 \%$ without hemorrhages.

TABLE 1. Clinical signs of ectopic pregnancy

\begin{tabular}{|c|c|c|}
\hline Clinical signs & $\begin{array}{c}\text { Number of } \\
\text { cases }\end{array}$ & Percentages \\
\hline Amenorrhea & 23 & $35.38 \%$ \\
\hline Pain & 60 & $92.30 \%$ \\
\hline Bleeding & 51 & $78.46 \%$ \\
\hline Lipothymia & 21 & $32.30 \%$ \\
\hline Palpable laterouterine formation & 45 & $69 \%$ \\
\hline Other signs & 10 & $15.38 \%$ \\
\hline
\end{tabular}

Most often, $65 \%$ of cases (42 patients), the evolution tends to complications, resulting in severe bleeding (by rupture of the fallopian tube or tuboabdominal abortion).

The investigations used to confirm the diagnosis of ectopic pregnancy in the Bucur Maternity Clinic are ultrasound and $\beta \mathrm{HCG}$ dosing.

Transvaginal ultrasound was used as a means of investigation for all patients in this study. With its help it was possible to establish the diagnosis of tubal pregnancy in the early stages, allowing a conservative intervention before the rupture of the fallopian tube.

The initial value of $\beta$ HCG in the studied group ranged from 500 to $3,880 \mathrm{mIU} / \mathrm{ml}$ (except for cervical pregnancies and those implanted on the postoperative cesarean scar, where the values were significantly higher, between 10,000 and $28,000 \mathrm{mUl} / \mathrm{ml}$ ), with increases in $\beta$ HCG values, at the second dosage, less than $35 \%$ (in the cases that allowed the re-evaluation).

Secondary to hemorrhagic complications (hemoperitoneum) there are changes in hematological and hemodynamic status, respectively: hemoglobin $(\mathrm{Hb})$, hematocrit $(\mathrm{Ht})$, blood pressure, pulse.

At the level of the study group, the range of $\mathrm{Hb}$ values is limited to $6.5 \mathrm{~g} / \mathrm{dl}$ (in severe cases, with severe hemorrhagic shock), respectively 3 patients, and $13 \mathrm{~g} / \mathrm{dl}$ (cases that were diagnosed in time, before complications), respectively 5 patients. The average value of $\mathrm{Hb}$, for all 65 patients, is $11 \mathrm{~g} / \mathrm{dl}$.

Simultaneously with the decrease of hemoglobin due to massive hemorrhage, there are also changes in $\mathrm{Ht}$, the range in which $\mathrm{Ht}$ values are included, in the case of patients in the study group, is between 25 and $45 \%$, the values being inversely proportional to the severity of the case. The average value of hematocrit is $35 \%$

For the monitoring and early diagnosis of hemorrhagic shock, in addition to the biological samples collected, we will use two clinical parameters: monitoring of blood pressure and ventricular allure, which very faithfully reflect changes in hemodynamic status, secondary to intraperitoneal hemorrhage.

Regardless of the value of systolic blood pressure in non-pathological conditions, a value of less than 80 $\mathrm{mmHg}$, corroborated with an increase in ventricular allure value of more than 100 beats/minute, reflects the existence of a hemorrhagic complication in the course of ectopic pregnancy.

Medical therapy with methotrexate has proven cost-effective compared to surgical therapy, the treatment being less traumatic for the patient and cheaper (reduces hospitalization), eliminates the risk of anesthesia and surgery, avoids possible tubal injuries while maintaining future fertility. In order to benefit from this treatment, patients are carefully selected based on clear criteria, following the indications and contraindications mentioned in the National Clinical Guidelines for Obstetrics and Gynecology regarding ectopic pregnancy.

At present, parenteral administration, by single injection of $50 \mathrm{mg} / \mathrm{m}^{2}$, without the administration of Leucovorin is usually used. The dose may be repeated if on day 7 BHCG did not decrease by more than $15 \%$ from day 4.

Before starting treatment, it is mandatory to collect a complete set of tests including blood count, serum creatinine, liver markers, $\beta$ HCG, blood group and Rh, both to determine the contraindications of administration and for subsequent monitoring during treatment.

Out of a total of 65 patients diagnosed with ectopic pregnancy 19 of the cases were treated with Metrotrexate $(29 \%) ; 45$ of the cases $(69 \%)$ benefited from surgical treatment; in 3 of the cases after the treatment with the established methotrexate, the surgical intervention was also practiced. In 2 of the cases, respectively the cervical pregnancies, after the treatment with the established methotrexate, the aspiration of the pregnancy was practiced, with the installation in one of the cases at the level of the endocolus of the hemostatic balloon. In 2 cases, respectively the pregnancies inserted on the scars after the cesarean section, after the treatment with methotrexate, the evacuation of the pregnancy was practiced, in one case by aspiration. In one case was diagnosed with ectopic pregnancy stopped in evolution, being only monitored, without the establishment of surgical or drug treatment.

At the level of the studied group, 13 of the patients who received medical treatment with methotrexate presented in the first 24-48 hours adverse reactions represented by nausea, vomiting and pelvic-abdominal pain, accompanied by quantitatively reduced metrorrhagia, and the $\beta$ HCG value at dosing after the first administration of methotrexate was increased in 11 of the 19 patients. 
Depending on the response to methotrexate treatment, the following were found: 14 cases received a dose of methotrexate with a decrease in serum $\beta$ HCG, one case requiring surgery ( $\beta$ HCG between $500 \mathrm{IU} / \mathrm{I}$ and $3500 \mathrm{IU} / \mathrm{I}) ; 2$ cases that received a dose of methotrexate had an increase in serum $\beta$ HCG levels, one case requiring surgery; 3 cases received 2 doses of methotrexate with a decrease in serum $\beta$ HCG levels after the second dose, one case requiring surgery.

In the studied group, out of 65 patients with ectopic pregnancies, a number of 2 presented pregnancies implanted on the post-cesarean scar representing a percentage of $3 \%$ of total ectopic pregnancies and $11 \%$ of total ectopic pregnancies with a history of cesarean section. These patients received single-dose systemic methotrexate in combination with intrasacular injection of methotrexate, by transcervical approach, under ultrasound guidance, with a favorable follow-up. Patients were followed by $\beta$ HCG dosing and ultrasound examination, pregnancy evacuation under intravenous anesthesia was performed, with subsequent monitoring of the uterine cavity ultrasound, monitoring with $\beta H C G$ decrease at 0 postoperatively.

Due to the teratogenic risk of methotrexate, it is recommended to use a method of contraception for at least 6 months.

All patients in the study had a $\beta$ HCG value of less than 5,000 IU// before methotrexate.

TABLE 2. Distribution of patients according to evolution

\begin{tabular}{|c|c|c|}
\hline Management & $\begin{array}{c}\text { Number of } \\
\text { cases }\end{array}$ & Percentage \\
\hline Methotrexate treatment & 16 & $25 \%$ \\
\hline Surgery & 42 & $65 \%$ \\
\hline Methotrexate and surgery & 3 & $4 \%$ \\
\hline Pregnancy stopped evolving & 1 & $2 \%$ \\
\hline Pregnancy aspiration & 3 & $4 \%$ \\
\hline
\end{tabular}

There are situations in which other types of more extensive radical interventions have been performed, these being imposed by the particularities of each case: the patient's age, damage to the tube by repeated inflammatory processes, the presence of complications etc.

From the studied group, 29 of the patients were laparotomized (64\%) and 16 (36\%) underwent laparoscopic interventions. Partial salpingectomy (27 patients), total salpingectomy (14 patients), annexectomy (2 patients), salpingostomy (2 patients) were performed.

The analysis of the obtained data shows that conservative surgical treatment was performed in only two cases, the rest of the patients being subjected to radical interventions determined by the surgical urgency (hemoperitoneum associated or not with hemorrhagic shock), previous tubal lesions, uncontrolled bleeding and the patient's desire expression for her reproductive future.

The therapeutic management of ectopic pregnancies diagnosed in patients in the study group is mainly surgical (69\% of patients), followed by medical management (25\%), pregnancy aspiration (4\%) and expectation management (2\%).

Another element to be evaluated in our study was the duration of hospitalization of patients, depending on the surgical method of intervention (laparoscopy versus laparotomy).

TABLE 3. The period of hospitalization of cases operated laparoscopically

\begin{tabular}{|c|c|c|}
\hline $\begin{array}{c}\text { Hospitalization } \\
\text { period (days) }\end{array}$ & $\begin{array}{c}\text { No. of laparoscopically } \\
\text { operated cases }\end{array}$ & Percentage \\
\hline $2-3$ & 11 & $68.75 \%$ \\
\hline $3-5$ & 4 & $25 \%$ \\
\hline$>5$ & 1 & $6.25 \%$ \\
\hline Total & 16 & $100 \%$ \\
\hline
\end{tabular}

We can conclude that the extension of the hospitalization period over 5 days is an exception, and is probably due to the occurrence of postoperative complications (severe headache after spinal anesthesia).

In the case of patients who underwent laparotomy, they had a hospitalization period of 5-9 days .

TABLE 4. The period of hospitalization of cases operated by laparotomy

\begin{tabular}{|c|c|c|}
\hline $\begin{array}{c}\text { Hospitalization } \\
\text { period (days) }\end{array}$ & $\begin{array}{c}\text { Number of cases } \\
\text { operated by laparotomy }\end{array}$ & Percentage \\
\hline 5-7 days & 24 & $82.75 \%$ \\
\hline $7-9$ days & 4 & $13.79 \%$ \\
\hline$>9$ days & 1 & $3.46 \%$ \\
\hline Total & 29 & $100 \%$ \\
\hline
\end{tabular}

Comparing the results, it can be concluded that laparoscopy significantly reduces the hospitalization period, contributing decisively to the rapid reintegration into society, increasing patient comfort, an excellent mental tone that promotes earlier healing, the number of complications decreases.

Among the complications occurred in the southern group we mention: hemoperitoneum present in 16 situations, hematocele encountered in 12 cases, hematosalpinx in 14 cases, in 2 cases the state of hemorrhagic shock appeared.

The study group included 2 patients with Rh negative, without isoimmunization, who were given anti-D immunoglobulin.

\section{DISCUSSIONS}

The discussions are generated following the comparative study carried out between the group of evalu- 
ated patients hospitalized in Bucur Maternity and the specialized literature.

Supporting the diagnosis of ectopic pregnancy is made by correlating all diagnostic methods, the clinical diagnosis with the paraclinical one, none of the methods having maximum efficiency (16).

The management of ectopic pregnancies is complex. Medical management is a simple and effective option in hemodynamically stable patients, with minimal symptoms and no intraperitoneal fluid, with clear indications for administration. Regarding the routes of administration, methotrexate can be administered orally, intravenously, intramuscularly or injected directly into the gestational sac under laparoscopic control, in case of corneal, cervical, or implanted pregnancy on the postoperative cesarean scar $(17,18)$. At the level of the study group, the success rate was $84 \%$ (16 cases out of 19 treated), by successful treatment is meant the avoidance of surgery, statistically similar to the evaluations performed in other studies.

Management of cervical pregnancies can be medical or surgical, or a combination of these, dilation and curettage not being used as first-line treatment due to the increased risk of bleeding (19). In our group of 2 patients, after administration of methotrexate, followed by decreased $\beta$ HCG values, dilation and curettage were performed, one of the patients requiring the intracervical placement of a $30 \mathrm{ml}$ Foley catheter for hemostasis.

The doses of methotrexate mentioned above are below the doses of adverse reactions: anemia, leukopenia, renal failure, stomatitis, ulcerative hemorrhagic enterocolitis, both in our study and in the studies consulted. Patients receiving Metrotrexate may experience pelvic-abdominal pain in the first few days, nausea, vomiting, these cases being more likely to be therapeutically successful, compared to those cases without obvious symptoms (20). In our group, $68 \%$ of patients had pelvic-abdominal pain accompanied by nausea in the first 48 hours after administration, in accordance with the data from the specialized literature.

Surgery is indicated in patients with hemodynamically compromised or other clinical signs of ruptured ectopic pregnancy (intense pelvic-abdominal pain correlated with evidence of intra-abdominal hemorrhage) as well as patients with contraindications to medical treatment. Laparoscopy has gained substantial ground recently, occupying a very important place in the diagnostic and therapeutic arsenal. Also, the indications for laparoscopy are much broader today, being recommended even in cases of complications (21-23). Rupture of ectopic pregnancy is the usual evolution of it, which is manifested by massive hemorrhage, shock, disseminated intravascular coagulation and even death $(24,25)$. After salpingectomy but especially salpingostomy, it is necessary to monitor $\beta \mathrm{HCG}$, an increased value of which still indicates the presence of trophoblastic tissue, which requires the administration of methotrexate.

\section{CONCLUSIONS}

The increase in the incidence of ectopic pregnancy in recent years, with the serious compromise of the woman's obstetric future and the life-threatening complications caused by it, impose both the need for a correct and early diagnosis and effective treatment to save the patient's life and, on as conservative as possible, for maintaining fertility, the development of means of paraclinical diagnosis contributing decisively to the establishment of subsequent therapeutic conduct.

Establishing early diagnosis allows the initiation of drug treatment, conservative, or the use of minimally invasive, conservative surgical techniques, with a high success rate. This reduces the number of days of hospitalization and preserves the patient's fertility in the future.

\section{Conflict of interest: none declared \\ Financial support: none declared}

\section{REFERENCES}

1. Butts S, Sammel M, Hummel A, Chittams J, Barnhart K. Risk factors and clinical features of recurrent ectopic pregnancy: a case control study. Fertil Steril. 2003 Dec;80(6):1340-4.

2. Skjeldestad FE, Hadgu A, Eriksson N. Epidemiology of repeat ectopic pregnancy: a population-based prospective cohort study. Obstet Gynecol. 1998 Jan;91(1):129-35.

3. Barnhart KT. Clinical practice. Ectopic pregnancy. N Engl J Med. 2009 Jul 23;361(4):379-87.

4. Fukami T, Emoto M, Tamura R, Kawarabayashi T. Sonographic findings of transvaginal color Doppler ultrasound in ectopic pregnancy. J Med Ultrason (2001). 2006 Mar;33(1):37-42.

5. Jurkovic D, Hillaby K, Woelfer B, Lawrence A, Salim R, Elson CJ. First-trimester diagnosis and management of pregnancies implanted into the lower uterine segment Cesarean section scar. Ultrasound Obstet Gynecol. 2003 Mar;21(3):220-7.

6. Seow KM, Huang LW, Lin YH, Lin MY, Tsai YL, Hwang JL. Cesarean scar pregnancy: issues in management. Ultrasound Obstet Gynecol. 2004 Mar;23(3):247-53.
7. Ades A, Parghi S. Laparoscopic Resection of Cesarean Scar Ectopic Pregnancy. J Minim Invasive Gynecol. 2017 May-Jun; 24(4):533-535.

8. Esther LSY, Chew KT, Rahman RA, Zainuddin AA, Hing EY, Kampan N. Uterine-conserving approach in ruptured intramyometrial ectopic pregnancy. Horm Mol Biol Clin Investig. 2020 Mar 13;41(2).

9. Hajenius PJ, Mol F, Mol BW, Bossuyt PM, Ankum WM, van der Veen F. Interventions for tubal ectopic pregnancy. Cochrane Database Syst Rev. 2007 Jan 24;2007(1):CD000324. 
10. Liu W, Shen L, Wang Q, Wang W, Sun Z. Uterine artery embolization combined with curettage vs. methotrexate plus curettage for cesarean scar pregnancy. Arch Gynecol Obstet. 2016 Jul;294(1):71-6.

11. Gao L, Hou YY, Sun F, Xia W, Yang Y, Tian $\mathrm{T}$, Chen $\mathrm{QF}$, Li XC. A retrospective comparative study evaluating the efficacy of adding intra-arterial methotrexate infusion to uterine artery embolisation followed by curettage for cesarean scar pregnancy. Arch Gynecol Obstet. 2018 May; 297(5):1205-1211.

12. Yang $H$, Li S, Ma Z, Jia Y. Therapeutic effects of uterine artery embolisation (UAE) and methotrexate (MTX) conservative therapy used in treatment of cesarean scar pregnancy. Arch Gynecol Obstet. 2016 Apr;293(4):819-23.

13. Lipscomb GH, Givens VM, Meyer NL, Bran D. Comparison of multidose and single-dose methotrexate protocols for the treatment of ectopic pregnancy. Am J Obstet Gynecol. 2005 Jun;192(6):1844-8.

14. Weibel HS, Alserri A, Reinhold C, Tulandi T. Multidose methotrexate treatment of cervical pregnancy. J Obstet Gynaecol Can. 2012 Apr;34(4):359-362.

15. Yeko TR, Mayer JC, Parsons AK, Maroulis GB. A prospective series of unruptured ectopic pregnancies treated by tubal injection with hyperosmolar glucose. Obstet Gynecol. 1995 Feb;85(2):265-8.

16. Taran FA, Kagan KO, Hübner M, Hoopmann M, Wallwiener D, Brucker S. The Diagnosis and Treatment of Ectopic Pregnancy. Dtsch Arztebl Int. 2015 Oct 9;112(41):693-705.

17. Akira S, Negishi Y, Abe T, Ichikawa M, Takeshita T. Prophylactic intratubal injection of methotrexate after linear salpingostomy for prevention of persistent ectopic pregnancy. J Obstet Gynaecol Res. 2008 Oct;34(5):885-9.

18. Mesogitis S, Pilalis A, Daskalakis G, Papantoniou N, Antsaklis A. Management of early viable cervical pregnancy. BJOG. 2005 Apr;112(4):409-11.

19. Reyftmann L, Vernhet $H$, Boulot $P$. Management of massive uterine bleeding in a cesarean scar pregnancy. Int J Gynaecol Obstet. 2005 May;89(2):154-5.
20. Murji A, Garbedian K, Thomas J, Cruickshank B. Conservative Management of Cervical Ectopic Pregnancy. J Obstet Gynaecol Can. 2015 Nov;37(11):1016-20.

21. Fylstra DL. Cervical pregnancy: 13 cases treated with suction curettage and balloon tamponade. Am J Obstet Gynecol. 2014 Jun;210(6):581.e1-5.

22. Habek D, Prka M. Preservational procedure of cervical pregnancy. Am J Obstet Gynecol. 2015 Jan;212(1):119.

23. Panelli DM, Phillips $\mathrm{CH}$, Brady PC. Incidence, diagnosis and management of tubal and nontubal ectopic pregnancies: a review. Fertil Res Pract. 2015 Oct 15;1:15.

24. Reyftmann $L$, Vernhet $H$, Boulot $P$. Management of massive uterine bleeding in a cesarean scar pregnancy. Int $J$ Gynaecol Obstet. 2005 May;89(2):154-5.

25. Liu S, Durai S. Management of a case of caesarean scar pregnancy and all its complications. BMJ Case Rep. 2016 May 17;2016:bcr2016215111. 